\title{
Editorial Mental health of doctors
}

\section{Jeremy Holmes}

Doctors, individually and collectively, are notoriously neglectful of their own health. Recently, this problem has been more openly discussed (Lens \& van der Wal, 1997) and several preventive programmes have been targeted at sick physicians (e.g. Pullen et al, 1994). At first sight this may appear self-indulgent. Doctors belong to a privileged sector within society which, in the main, enjoys comparatively good income, security of employment, high status and satisfying work. From an epidemiological perspective, the general health of doctors is likely to be far better than that of many other occupational groups. Why focus specifically on physicians, when bus drivers, unskilled labourers, single working mothers and the unemployed are so much more at risk?

\section{Why doctors are at risk}

There are several answers to this question. The first, which forms the main subject of this special issue of Advances in Psychiatric Treatment, is that while the physical health of doctors may be superior to that of the general population, the same is not true of their mental health. The ill-health of doctors is mainly psychiatric ill health, and doctors are more prone to anxiety/depression, suicide and alcohol and substance misuse than comparable occupational groups. The central issue of psychiatric morbidity in doctors compounds the problems of the recursiveness of doctors treating other doctors and the general suspicion and prejudice about psychiatric disorder within society. If we cannot overcome our own reluctance to face and accept the reality of psychological illness, what hope is there for the rest of society?
A further justification follows from this. Doctors form a test case of their own preventive strategies. The classic example here was Doll's demonstration of the relationship between smoking and lung cancer. Doll's findings were appreciated early on by the medical profession and the preventive effects of stopping smoking were first illustrated by the changing smoking habits of doctors.

Why, then, do doctors make such bad patients? Many doctors are reluctant to consult their general practitioner about physical complaints, and this avoidance is strengthened when the difficulty is psychological. There are several factors underlying this resistance, but from a psychodynamic perspective perhaps the most important is the process by which doctors project their weakness and vulnerability onto their patients. This may help us to feel stronger and more powerful ourselves, and fosters the fantasy that our every need and desire can be met if only the right solution can be found. This omnipotent defence is reinforced by the social structure of medical careers, and especially the inherent competitiveness of hospital medicine, and is often shattered by first exposure to the ambivalence and uncertainty that is inherent in the practice of self-aware psychiatry.

Like all defences, this projective process has its advantages and handicaps. When all is going well, denial of vulnerability adds to doctors' charisma - which benefits us and our patients. But in a changing world, where doctors' powers are under threat and at the same time more and more is demanded of them, the Faustian pact becomes unstable. If a doctor is all-powerful and invulnerable, what is he or she to do when doubt, depression, divorce or difficulty strikes? Physicians are supposed to heal themselves - hence the resort to alcohol, drugs, compulsive working and denial, each of which adds one more twist to the vicious 
spiral. Many doctors are not registered with a general practitioner, and, when they are, it is often a close colleague with whom they would find it impossible to discuss intimate matters. Here the traditional lack of boundaries in doctors' lives between private and public, between one's self and one's work, often flying under the banner of 'dedication' - becomes another handicap to effective medical care. All this is compounded by the stigma of mental illness, and the widespread fear and ignorance, even within medical circles, of psychological difficulty. To sit, as I have done, on an appointments committee and see an excellent candidate rejected because of a prior history of manic depression is a profoundly saddening experience for a psychiatrist, especially if contrasted with the response (admiration at a person's fortitude and ability to overcome difficulty) which a physical illness of comparable severity can evoke. The courageous self-revelations of Jamison (1996), who is both a professor of psychiatry and a manic depression sufferer, may go some way to redress these kinds of prejudicial attitudes.

\section{Prevention of psychiatric morbidity and the causes of stress}

If we are to take seriously the project of a preventive strategy for psychological health in doctors the lessons of preventive medicine need to be studied carefully. Doll's work depended on finding an external factor responsible for a defined pathology. The factor most often cited as responsible for high levels of psychiatric morbidity among doctors is stress. What is it about medical work that is stressful, and how can this be reduced without jeopardising the nature of the work? If answers could be found to these questions, here too the health of doctors could be exemplary.

Some of the sources of stress in medicine are self-evident, for example the unsociable hours and life-and-death responsibility inherent in the job. Others are all too familiar to doctors themselves inflexible and heirarchical career patterns, intolerance of non-conformity, and a generally 'macho' culture often sequestered from everyday life. Others are more subtle. There is good evidence to suggest a relationship between occupational control and stress - the less one feels in control of one's working environment, the more stressful it becomes. The health service 'reforms' of the past decade have certainly shifted power and control away from doctors, whose sense of being in charge of their own destiny has correspondingly diminished. Wilkinson's (1996) recent work on relative deprivation and health may also be relevant here. Social inequality, rather than absolute poverty, seems to determine the health of populations. Psychological ill-health in doctors may be a symptom of a wider problem of increasing social inequity and powerlessness. It remains to be seen whether the new Labour government can start to reverse these feelings of helplessness and marginalisation.

Another potential source of stress lies in the personality characteristics of doctors themselves. This follows in part from the impulses that drive people to chose medicine as a career, partly from the selection processes for medical schools, and partly the developmental experience of becoming a doctor. All centre around the combination of robustness and sensitivity that makes for good doctoring, and the paradox that in order to relieve suffering one has to expose oneself to it - the wounded healer has to learn neither to succumb to his pain, nor to turn his back on it. The oversensitive are liable to respond by over-identification and over-involvement with their patients and become compulsive carers, while at the opposite pole are those who react to suffering by denial or avoidance or compulsive working. Becoming aware of these patterns can be a helpful first step towards a more balanced pattern of work, and an important role for psychiatrists and psychotherapists may lie in increasing such awareness.

\section{The medical profession's duty to heal itself}

For all its contemporary ills, medicine remains a vocation. It is more than a reasonably congenial way of earning a living, although there is no reason why it should not be that too. The medical profession aims not only to help its patients, but also to be a force for good within society. The establishment of the National Health Service by the post-war Labour government was one of the highest expressions of that ideal. Just as society expects, or should expect, its priests to live ethically or its architects to live in visually pleasing environments, so doctors have a duty to attend to their own health.

At an individual level, 'physician heal thyself' is an injunction that promotes ill-health and should be rejected, but self-understanding is a first step towards self-healing. At a collective level it is a challenge which the profession is at last beginning 
to take seriously. This isssue of Advances in Psychiatric Treatment reflects the impressive contribution psychiatry can make towards improved health among doctors, both for their own and their patients' sakes. The key issue is a change in the milieu of medicine. The dialogue between social psychiatry and psychotherapy which is evident in these contributions is a signpost for medicine in the next century, which the profession will ignore at its peril.

\section{References}

Jamison, K. (1996) An Unquiet Mind: A Memoir of Moods and Madness. London: Picador.

Lens, P., van der Wal, G. (1997) Problem Doctors: A Conspiracy of Silence. Amsterdam: JOS.

Pullen, D., Lonie, C., Lyle, D., et al (1994) Medical care of doctors. Medical Journal of Australia, 163, 481-484.

Wilkinson, R. (1996) Unhealthy Societies: The Afflictions of Inequality. London: Routledge. 